\title{
Development and Exploratory Analysis of Software to Detect Look-Alike, Sound-Alike Medicine Names
}

Running title: Software to Detect LASA Medicine Names

Lynne Emmerton, PhD MPS ${ }^{a *}$

Colin Curtain, PhD MPS

Girish Swaminathan, MHSM GradDip(ClinPharm) FISQua ${ }^{c}$

Helen Dowling, BPharm GradDipQIHCare DipHospPharm(Admin) MFIP AFAIM FSHP

a School of Pharmacy and Biomedical Sciences

Curtin University

GPO Box U1987

Perth WA 6845

Australia

lynne.emmerton@curtin.edu.au

b Discipline of Pharmacy, School of Medicine

University of Tasmania

Private Bag 34

Hobart Tas 7000

Australia

colin.curtain@utas.edu.au

c Australian Commission on Safety and Quality in Health Care

GPO Box 5480

Sydney NSW 2001

Australia

girish.swaminathan@ safetyandquality.gov.au

helen.dowling@safetyandquality.gov.au

*Author for correspondence

Key words: Medicine, Look-alike sound-alike, Similarity, Risk, Safety, Software

Word count: 3212 (excluding abstract, tables, figure, required statements and references) 


\title{
Development and Exploratory Analysis of Software to Detect \\ Look-Alike, Sound-Alike Medicine Names
}

\begin{abstract}
Background. 'Look-alike, sound-alike' (LASA) medicines may be confused by prescribers, pharmacists, nurses and patients, with serious consequences for patient safety. The current research aimed to develop and trial software to proactively identify LASA medicines by computing medicine name similarity scores.
\end{abstract}

Methods. Literature review identified open-source software from the United States Food and Drug Administration for screening of proposed medicine names. We adapted and refined this software to compute similarity scores (0.0000-1.0000) for all possible pairs of medicines registered in Australia. Two-fold exploratory analysis compared:

1. Computed similarity scores vs manually-calculated similarity scores that had used a different algorithm and underpinned development of Australia's 2011 Tall Man Lettering List ('the 2011 List')

2. Computed risk category vs expert-consensus risk category that underpinned the 2011 List. Results. Screening of the Australian medicines register identified 7,750 medicine pairs with at least moderate (arbitrarily $\geq 0.6600$ ) name similarity, including many oncology, immunomodulating and neuromuscular-blocking medicines. Computed similarity scores and resulting risk categories demonstrated a modest correlation with the manually-calculated similarity scores $(r=0.324, \mathrm{p}<0.002,95 \%$ CI 0.119-0.529). However, agreement between the resulting risk categories was not significant (Cohen's kappa $=-0.162$, standard error $=0.063$. 
Conclusions. The software ( $L A S A v 2)$ has potential to identify pairs of confusable medicines. It is recommended to supplement incident reports in risk-management programs, and to facilitate pre-screening of medicine names prior to brand/trade name approval and inclusion of medicines in formularies.

Key words: Medicine, Look-alike sound-alike, Similarity, Risk, Safety, Software

\section{INTRODUCTION}

Risks associated with confusable medicine names have been recognized in the literature ${ }^{1-4}$ and the emergence of quality and safety initiatives in the past decade. Medicine names that look alike and/or sound alike (LASA) require vigilance during their prescribing, dispensing, administration to patients and monitoring, ${ }^{1}$ and across manual and electronic processes. ${ }^{5}$

Internationally, marketing approval for new medicines includes consideration of the uniqueness of the medicine name. ${ }^{6-9}$ However, it is evident from clinical incident reports and characterization of risks that name approval processes are insufficient to prevent LASA errors. ${ }^{2,3,10}$ Numerous safety initiatives, generally based on drawing attention to medicines at risk of being confused, have been introduced in clinical practice to reduce erroneous substitution of a LASA medicine. ${ }^{4,5}$

One such initiative is the 2011 National Tall Man Lettering List ('the 2011 List') published by the Australian Commission on Safety and Quality in Health Care, and informed by Australian and international reports of errors and near misses involving LASA medicines. ${ }^{11}$ Use of Tall Man lettering in the 2011 List aims to draw attention to a syllable or string of 
letters that distinguishes one medicine name from a similar name. ${ }^{11-16}$ Derivation of the 2011 List was as follows (Table 1): firstly, a list of LASA medicine pairs was compiled from international and local reports involving confusable medicines, in the absence of a national repository of LASA error reports; secondly, a novel risk matrix was produced to map the likelihood of confusion of these name pairs in clinical practice, and the clinical consequence if they were indeed switched, which produced a risk classification for this confusion; and thirdly, medicine pairs with the highest risk classifications were presented in mid-Tall Man convention in the 2011 List. $^{17}$ 


\begin{tabular}{|c|c|c|}
\hline Stage & Source(s)/Reference(s) & Output \\
\hline $\begin{array}{l}\text { Systematic } \\
\text { compilation of } \\
\text { an Australian } \\
\text { list of LASA } \\
\text { medicine } \\
\text { names } \\
\end{array}$ & $\begin{array}{l}\text { 1. Australian medicines in international lists of } \\
\text { confusable medicines } \\
\text { 2. Error reports from medication safety agencies } \\
\text { 3. Jurisdictional LASA incident databases } \\
\text { 4. Online LASA warnings and alerts in the } \\
\text { 'grey' literature }\end{array}$ & $\begin{array}{l}341 \text { unique medicine } \\
\text { names } \\
(156 \text { generic }+185 \\
\text { proprietary medicines })\end{array}$ \\
\hline \multirow[t]{2}{*}{$\begin{array}{l}\text { Prioritisation } \\
\text { of drug name } \\
\text { pairs based on } \\
\text { their risk for } \\
\text { confusion and } \\
\text { consequences } \\
\text { of this type of } \\
\text { error }\end{array}$} & $\begin{array}{l}\text { Risk matrix utilizing: } \\
\text { 1. Likelihood of confusion score out of } 100 \text { (later } \\
\text { classified in quintiles): } \\
\text { a. Name similarity calculated using an online } \\
\text { BI-SIM calculator }{ }^{\dagger}(70 \%) \text { (one-against-one) } \\
\text { b. Strength similarity }(0,10,20)(20 \%) \\
\text { c. Administration route similarity }(0,2.5,5) \\
(5 \%) \\
\text { d. Dosage form similarity }(0,2.5,5)(5 \%)\end{array}$ & $\begin{array}{l}\text { Average likelihood of } \\
\text { confusion score }=48.1 \\
\text { (range } 15.0-82.5 \text { ) }\end{array}$ \\
\hline & $\begin{array}{l}\text { 2. Consequences of confusion between the } \\
\text { similar medicines ('minimum', 'minor', } \\
\text { 'moderate', 'major', 'catastrophic'), based on } \\
\text { consensus between trios of experts, and } \\
\text { considering characteristics of the medicine and } \\
\text { number of doses of the wrong medicine that } \\
\text { would cause harm. Assumptions: } \\
\text { a. That exposure to the wrong medicine was } \\
\text { short term } \\
\text { b. That the patient was otherwise healthy } \\
\text { c. That the error was in the direction of greater } \\
\text { harm (A given instead of B, or B given } \\
\text { instead of A) }\end{array}$ & $\begin{array}{l}\text { Intra-class correlation } \\
\text { across } 20 \text { medicine pairs } \\
\text { rated by } 31 \text { experts = } \\
0.961 \\
23 \text { medicine pairs rated } \\
\text { by trios of } 28 \text { experts: } \\
\text { agreement for }>85 \% \text { of } \\
\text { medicine pairs }\end{array}$ \\
\hline $\begin{array}{l}\text { Derivation of } \\
\text { Tall Man } \\
\text { lettering for } \\
\text { the highest } \\
\text { priority pairs } \\
\text { of medicine } \\
\text { names }\end{array}$ & $\begin{array}{l}\text { Mid-Tall Man convention" for medicine name } \\
\text { pairs identified as 'extreme' or 'high' risk by the } \\
\text { risk matrix }\end{array}$ & $\begin{array}{l}\text { 'Extreme' risk category: } \\
35 \text { medicine pairs ( } 64 \\
\text { medicines) } \\
\text { Final Tall Man list }{ }^{\S}: 204 \\
\text { medicine names }\end{array}$ \\
\hline
\end{tabular}

$\dagger$ www.cs.toronto.edu/ aditya/strcmp2/

$¥$ Gerrett D, Gale A, Darker IT, Filik R, Purdy KJ. Final report of the use of Tall Man lettering to minimise selection errors of medicine names in computer prescribing and dispensing systems. London, UK: NHS Connecting for Health; 2009.

$\S$ Australian Commission on Safety and Quality in Health Care. National standard for the application of Tall Man lettering: project report. ACSQHC; 2011. http://www.safetyandquality.gov.au/wpcontent/uploads/2013/03/National-Standard-for-the-Application-of-Tall-Man-Lettering-Project-Report-withappendices-PDF-700KB.pdf.

Table 1. Derivation of the 2011 National Tall Man Lettering List 
While methodologically robust when benchmarked to international literature in 2016, the processes to produce and manually maintain the 2011 List have been resource intensive. Furthermore, reliance on incident reports for consideration of new LASA risks is limited by the likely under-reporting of incidents and under-recognition of LASA risks, the retrospective nature of these data, and an associated lag in preventing further errors or near misses. A more proactive, automated approach warrants investigation to supplement incident reports from clinical practice.

In the United States of America (USA), the Food and Drug Administration has published open-source software for pharmaceutical manufacturers to screen their proposed brand names prior to submission for approval. Known as Phonetic and Orthographic Computer Analysis (POCA), this software uses linguistics algorithms to compute composite similarity scores for pairs of medicine names. ${ }^{18}$ The composite score applies equal weighting between an orthographic (look-alike) similarity score (a mean of the BI-SIM and LED) and a phonetic (sound-alike) similarity score (ALINE), and is normalized to a 'perfect match' of $1.0000 .^{18}$ This algorithm is also applied in Health Canada's Drug Product Database Online Query, ${ }^{19}$ with sponsors required to submit a LASA brand name assessment for marketing approval. ${ }^{7}$ Key research in the field of LASA name similarity recommends a linear combination of measures, which is likely to outperform any individual measure. ${ }^{20}$ While the POCA produces an objective measure of name similarity, human processes explore additional risks for confusion through name simulation studies and consideration of similarity in the medicines' strength, dose, indication, dosing frequency and route of administration. ${ }^{18}$ 
The premise of the current research, which is novel in Australia, is that principles of the POCA software may be applied in Australia to proactively screen all approved medicine names to identify LASA medicines that should be prioritized for risk-management initiatives.

\section{MATERIALS AND METHODS}

The primary objective of this research was to determine the feasibility of producing an Australian adaptation of the POCA for proactive (automated) screening of LASA medicines. A second objective was an exploratory comparison of the resulting similarity scores and risk categories to the manually-calculated similarity scores and risk classifications that underpinned the 2011 List.

\subsection{Stage 1: Software development}

Development of the Australian software is reported in detail in Supplemental File 1. This stage involved: investigation of the POCA software; preparation of the source data, via transformation of the Australian Medicines Terminology database ${ }^{21}$ (representing the Australian Register of Therapeutic Goods, comprising approximately 80,000 products ${ }^{22}$ ); programming of BI-SIM, LED and ALINE algorithms for all-against-all screening of medicine name similarity; software development; testing and refinement of screening processes and outputs; and review of the thresholds for output scores. A user interface for $a d$ $h o c$ screening of medicine name similarity was also produced. Medicine names consisted of single-word trade names and generic names, with a minimum length of five alphabetic characters. 


\subsection{Stage 2: Review of outputs}

Output files were managed using Excel ${ }^{\circledR}$ for descriptive analysis. Various thresholds for 'extreme', 'high' and 'moderate' similarity (risk categories), guided by the POCA, ${ }^{18}$ were trialled to manage data volume. Sorting by medicine name was facilitated by presenting the medicine name pairs as Name A vs Name B, and Name B vs Name A, in a single column.

The medicine name pairs with manually-calculated similarity scores prepared for the 2011 List (Table 1) were mapped into the computed output file of name pairs with at least 'moderate' similarity scores, for analysis using SPSS ${ }^{\circledR}$. Correlation between name similarity scores (manual vs computed) was explored using the Pearson correlation coefficient. Agreement between the resulting risk categories was determined using Cohen's kappa. Significance was determined at $\mathrm{p}<0.05$.

Further descriptive analysis identified LASA medicines recognized as high risk according to the 'APINCH' acronym: antimicrobials, potassium and other electrolytes, insulin, narcotics and other sedatives, chemotherapeutic agents, heparin and other anticoagulants. ${ }^{23}$ This was considered important for an automated system to proactively identify pairs of medicines with greater clinical consequences if accidentally switched. A comprehensive list of APINCH medicines was not available; consequently, high-risk medicines (list available on request) were confirmed via published Medication Safety Alerts and Notices, ${ }^{24}$ state Health Department websites and the Australian Medicines Handbook. ${ }^{25}$

\section{RESULTS}




\subsection{Stage 1: Software development}

The code was initially produced in Python and converted to $\mathrm{C}++$ for operational efficiency (Supplemental File 1, "Identification and preparation of similarity algorithms"). For the purposes of this trial, the combined similarity score was an unweighted mean of the orthographic similarity score (BI-SIM and LED) and the phonetic similarity score (ALINE), as per the POCA algorithm. ${ }^{18}$

The initial run produced over 20 million comparisons (4,528 medicine names compared against each other), requiring over 50 hours of computational time. Removal of duplicate name pairs and self-matches then more than halved the computational load. Further refinements reduced the computational time to approximately 15 hours (Supplemental File 1, "Testing and refinement of the software output").

The final software and user interface was named $\boldsymbol{L A S A} \boldsymbol{v} 2$ : Look-alike sound-alike Automated Screening Application (Figure 1). 


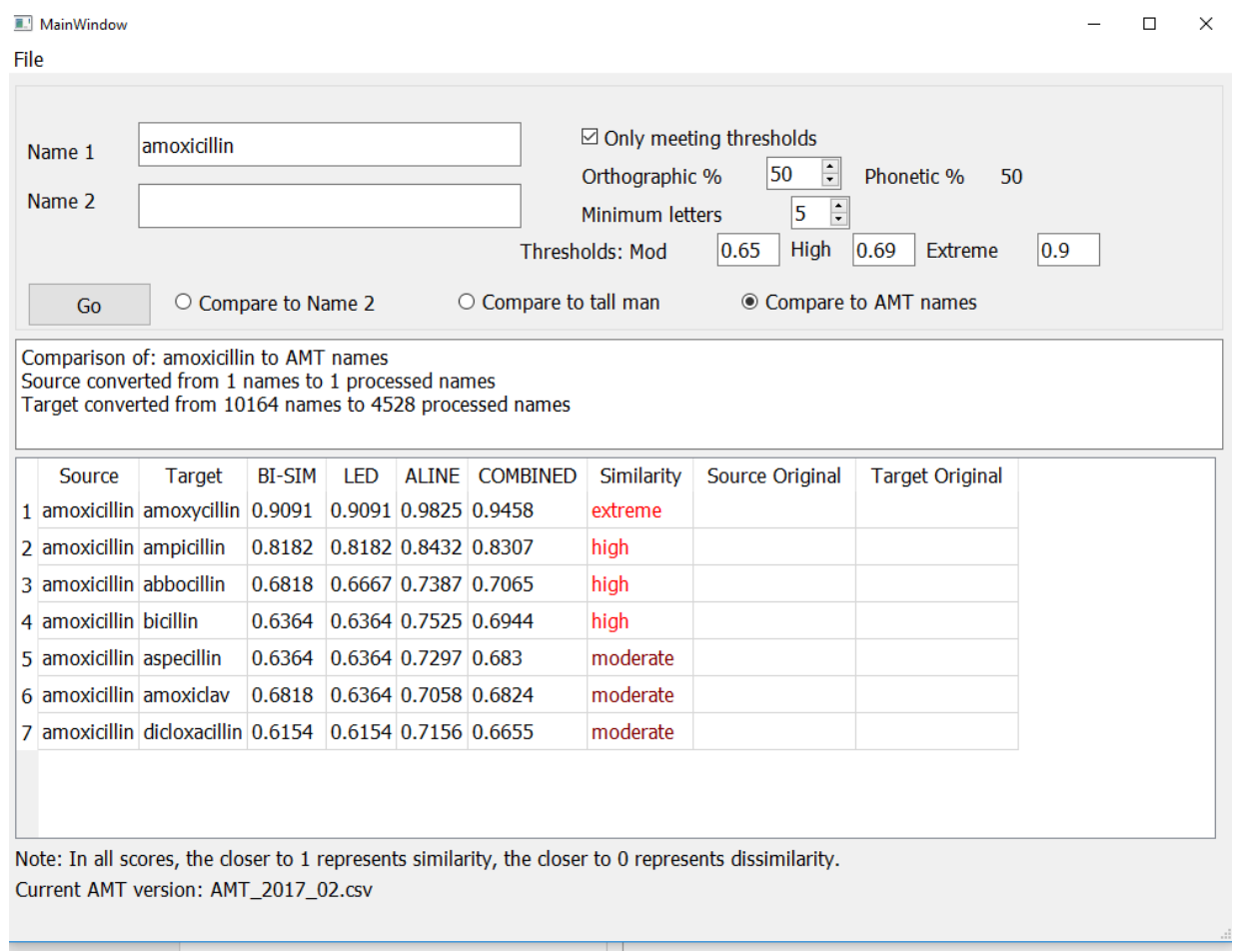

Figure 1. LASA $v 2$ interface

(color print not required)

\subsection{Stage 2: Review of outputs}

Trials of various thresholds for risk categories with respect to data volume, and visual inspection of the outputs for apparent name similarity, indicated an arbitrary threshold similarity score of 0.6600 would produce a comprehensive, but useful and manageable, database of similarity scores that could be used in conjunction with clinical risk considerations. Supplemental File Table 7 details the output volumes at various threshold settings.

The total number of LASA medicine name pairs with a computed similarity score of at least 'moderate' similarity (0.6600) was 7,750, excluding duplicates and self-paired names. The proposed category thresholds, which are practical variants of those used in the POCA, were: 
'moderate' similarity, defined as 0.6600-0.6899 (3,521 pairs); 'high' similarity, defined as 0.6900-0.8999 (4,195 pairs); and 'extreme' similarity, defined as $\geq 0.9000$ (34 pairs). The 34 name pairs with 'extreme' similarity are presented in Table 2.

\begin{tabular}{|lll|}
\hline $\begin{array}{l}\text { Computed } \\
\text { score }\end{array}$ & Name 1 & Name 2 \\
\hline 0.9605 & hexachlorophene & hexachlorophane \\
0.9521 & diethylamino & diethylamine \\
0.9516 & dlalphatocopheryl & dlalphatocopherol \\
0.9483 & dalphatocopheryl & dalphatocopherol \\
0.9463 & cyclosporin & ciclosporin \\
0.9458 & amoxycillin & amoxicillin \\
0.9356 & carbohydrates & carbohydrate \\
0.9356 & triglycerides & triglyceride \\
0.9338 & amoxyclav & amoxiclav \\
0.9332 & dlalphatocopherol & dalphatocopherol \\
0.9332 & dalphatocopherol & dlalphatocopherol \\
0.9271 & mitozantrone & mitoxantrone \\
0.9220 & oestradiol & estradiol \\
0.9210 & pentavite & centavite \\
0.9199 & benztropine & benzatropine \\
0.9195 & quipine & quepine \\
0.9180 & gentiana & gentian \\
0.9179 & pyrethrins & pyrethrin \\
0.9153 & sennosides & sennoside \\
0.9151 & glutose & glucose \\
0.9150 & taxoccord & tacoccord \\
0.9148 & glucagon & glucagen \\
0.9148 & sorbitol & sorbisol \\
0.9141 & moxiclav & amoxiclav \\
0.9125 & glucoboy & glucobay \\
0.9073 & glucocare & glucocard \\
0.9067 & acetic & acetec \\
0.9052 & temaccord & gemaccord \\
0.9034 & primaxin & primacin \\
0.9028 & panalgesic & analgesic \\
0.9025 & indomethacin & indometacin \\
0.9024 & mohexal & amohexal \\
0.9019 & mitomycin & minomycin \\
0.9004 & cocoamphodiacetic & cocoamphodiacetate \\
\hline & & \\
\hline
\end{tabular}

Table 2. Name pairs with 'extreme' computed similarity 
Many of these pairs suggest the computed scores have high sensitivity to spelling variations of the same medicine name (amoxycillin, the historical Australian spelling, vs amoxicillin, the internationally-recognised spelling). Within the 'extreme' risk category, the only medicine name pair that had appeared in the 2011 List was primacin (containing the antimalarial primaquine; Boucher \& Muir Pty Ltd, Sydney) vs primaxin (containing the antibacterial imipenem; Merck Sharp \& Dohme (Australia) Pty Ltd, Sydney), with a computed similarity score 0.9034 . The difference between the two names is a single similarsounding phoneme ('c' vs 'x'). The manually-calculated similarity for this pair in 2011 (following the method in Table 1) was modest (0.6125); however, this pair had been included in the 2011 List on account of the clinically-determined risk category.

Mapping of the manually-calculated similarity scores against the computed similarity scores (retained from $>0.6600$ for manageability of the outputs) identified 86 name pairs with both types of scores. The manually-calculated similarity scores for these 86 pairs ranged from 0.3222-0.8250, while the computed similarity scores ranged from 0.6609-0.9034. There was significant, but modest, correlation between the manual and computed scores for the 86 name pairs $(\mathrm{r}=0.324, \mathrm{p}<0.002,95 \%$ CI $0.119-0.529)$.

Exploratory comparison of computed risk categories and the manually-determined risk categories was possible for 84 name pairs in 'moderate', 'high' or 'extreme' categories (Table 3). The Cohen's kappa of -0.162 (standard error $=0.063)$ indicated no agreement between the ratings. 


\begin{tabular}{ll|ccc|c|} 
& & \multicolumn{3}{c}{ Manually-Determined Risk Rating } \\
& & Extreme & High & Moderate & \multicolumn{1}{c}{ Total } \\
\cline { 3 - 6 } & Extreme & 1 & 0 & 0 & 1 \\
Computed & High & 13 & 23 & 25 & 61 \\
Risk & Moderate & 8 & 13 & 1 & 22 \\
\cline { 3 - 6 } Rating & Total & 22 & 36 & 26 & 84 \\
\cline { 3 - 6 } & & & & &
\end{tabular}

Table 3. Comparison of manually-determined and computed risk ratings

Medicine name pairs of greatest concern were those where both names of the pair were identified as high-risk medicines according to the 'APINCH' acronym. There were no medicines in 'extremely' similar name pairs where both medicines were considered high risk. However, 183 medicine pairs with 'high' similarity scores, where either or both medicines were high risk, and 109 'moderate' similarity scores where either or both medicines were high risk, were identified. This is a conservative estimate due to the truncation of some medicine names for computation of their similarity scores.

Within the 183 highly-similar pairs, 127 were classified according to the Australian Medicines Handbook as Monoclonal Antibodies and Antineoplastics, ${ }^{25}$ mostly with suffixes 'nib' and 'mab'. The potential for LASA confusion within this group is evident from the following examples: eculizumab vs efalizumab (computed similarity score 0.8570), mepolizumab vs vedolizumab (0.8424) and belimumab vs golimumab (0.8336). 


\section{DISCUSSION}

Development of this LASA v2 software for use in Australia, to assess the similarity (or conversely, the uniqueness) of medicines names, appears to be feasible. The software is also potentially valuable for all-against-all screening of proprietary and generic medicines at a national level, to proactively identify medicines at risk of confusion in clinical practice. This work is novel in two respects. Firstly, the successful adaption of software developed to assess proposed medicine names in America, to screen a national register of medicine names in another country (Australia), suggests further international development is possible. Secondly, the use of automation to proactively identify medicine names at risk of confusion has not been previously reported as part of a national clinical safety initiative. While automated screening of databases of medication orders, diagnostic claims and drug indications has been trialled to identify potential errors may have involved LASA medicines, ${ }^{1,4}$ that approach was retrospective, requiring years of data to detect low-incidence potential errors. The algorithm was initially limited to the BI-SIM, a single measure of orthographic similarity, then extended to a mean of 10 similarity measures, suggestive of the lack of consensus between authors in this field of linguistics. Real-time application of that method would require accurate diagnostic data and machine learning to apply and adapt the risk criteria.

Limitations of the current work, in terms of the specificity of the software, predominantly rest with the inherent differences between a) the manual protocol to identify medicine name pairs at risk of confusion and b) the automated system computing similarity scores for pairs of medicine names. The automated system was not intended to replicate the manual system, as it engages large-scale screening of name similarity without the capacity for expert opinion, and a risk classification based on name similarity alone. Furthermore, the manual system was not 
considered a 'gold standard', merely the system that had served to establish the 2011 List but lacked proactivity and efficiency. The comparison between the systems was exploratory, and we have provided commentary around the feasibility of the automated system for maintenance of the 2011 List.

A second limitation is the transformations of the Australian Medicines Terminology database required for the computation of similarity scores ${ }^{21}$ Multi-word names were truncated to the first word, which resulted in retention of some irregular medicine names (e.g. 'vitamin' or 'protein') requiring screening out, yet retained extensive names, provided they comprised at least five alpha characters.

Since April 2016, Australia has been transitioning to international nomenclature for spelling of generic medicine names. ${ }^{26}$ During the transition period, where the Australian Medicines Terminology retains both the traditional and international spelling (e.g. cyclosporine and ciclosporin), these variations of the same name are likely to remain amongst the highest similarity scores. Ostensibly, these serve as a positive control in review of the computed scores. The current analysis requires manual elimination of these data to reduce the output volume.

It was beyond the scope of this work to explore alternative measures of orthographic and phonetic medicine name similarity. The component measures - BI-SIM and LED for measurement of look-alike similarity, and ALINE for measurement of sound-alike similarity ${ }^{18}$ - have been established in the linguistics literature. Literature also supports the combined use of these measures, ${ }^{20}$ in light of a large body of evidence reporting errors of medicine confusion that had resulted from medicine names erroneously read (or recognized 
from lists) and/or heard. ${ }^{27}$ For the purposes of this trial, orthographic and phonetic measures were equally weighted, as applied in the POCA $;{ }^{18}$ however, our software interface enables manipulation of their relative weighting for relevance to particular clinical practice settings.

Manually-calculated similarity scores in the 2011 List only applied the BI-SIM, along with an arbitrary algorithm requiring human determination of the similarity in dosage forms, routes of administration and strengths of the LASA medicine pairs. ${ }^{11}$ Due to the computational load in our testing, Australian Medicines Terminology data fields describing the medicine formulation and strength were eliminated. More recent research into predictors of confusable medicine pairs by Her and Toh confirmed that similarity in product characteristics, particularly between medicines from the same manufacturer, are significant. ${ }^{3}$ Our premise is that these factors may be considered by clinical experts alongside practice-related risks such as the proximity of storage of the LASA medicines and their use in high-pressure clinical environments. As such, the computational outputs require interpretation in relation to the clinical setting and the respective presentations of the confused medicines.

The current description of the computational output, followed by comparison of the computed composite similarity scores to the manually-calculated scores, was a pragmatic method to investigate the feasibility of the automated screening. The modest correlation between the computed and manually-calculated similarity scores highlights the potential of the automated method. This correlation could be due in part to both methods including in their calculation the BI-SIM measure of orthographic (look-alike) name similarity. However, the requirement for cognitive input in the manual calculation of similarity scores was the underlying reason for introduction of the automated screening. 
The lack of notable agreement between risk categories (using Cohen's kappa) highlights the methodological differences in how the categories were generated: the computed risk category was determined entirely on similarity scores, while the manually-calculated risk category included clinical judgment of the consequences to the patient if the two medicines were accidentally switched. We argue that there is still a place for clinical judgment in assessment of LASA medicines, but reserved for interpretation of data for the highest-risk medicine pairs.

This exploratory analysis would be enhanced by ongoing extraction of data from clinical incident report databases, as well as cognitive tests in the method of Schroeder and colleagues,${ }^{28}$ whereby health professionals perform medicine name recognition tasks under controlled conditions. It should be recognized that clinical incidents relating to LASA medicines may also involve name pairs with low computed similarity, but other risks for confusion (e.g. alphabetical proximity ${ }^{5}$ ) that should be alerted to healthcare staff to improve their familiarity with medicines. As such, the current research recommends retention of error and near-miss reporting to draw attention to medicine safety issues in clinical practice, to inform educational initiatives, and to supplement automated screening of medicine name similarity.

This work has potential for use in Australia's pre-approval screening of the uniqueness of medicine brand/trade names, either placing the onus on the sponsor (pharmaceutical company) to provide this evidence, as is the case in the $\mathrm{USA}^{18}$ and Canada, ${ }^{7}$ or performed by Australia's respective medicines regulatory body. While the potential introduction of a similar approach in Australia represents a longer-term strategy to reduce LASA medicine risks, it may avert further infiltration of the market with confusable medicine names. 
Regardless of formal adoption in pre-approval processes, we recommend regular updates of our computation to screen newly-approved medicines against the existing market to identify current risks. This would also eliminate discontinued medicines that are no longer relevant for clinical alerts within decision-support systems, ${ }^{5,16}$ and maintain the database with minimal computational load. It was beyond the scope of the current work to apply the software to predict LASA medicine errors in clinical practice; other bodies of research have attempted this using algorithms designed to search for anomalies within electronic medication records. ${ }^{1,428}$ Our software is intended for use by regulatory and safety authorities in medicines governance.

Further research is underway into medicine name confusion in high-risk (APINCH) medicine groups. A number of such groups, e.g. Monoclonal Antibodies and Antineoplastics, have seen significant increases in their number of medicines and complexity of their names, and subsequent risk for LASA errors. Of note is recent work in an American cancer centre to differentiate LASA antineoplastic agents throughout all stages of the centre's medication management system. ${ }^{29}$ Specifically, alerts for selected medicine pairs may be integrated into prescribing and dispensing software through direct use of Tall Man lettering ${ }^{5}$ or pop-up alerts ${ }^{16}$ either applying a national standard ${ }^{11}$ or more specific list tailored to the clinical speciality.

In conclusion, medicines safety initiatives and risk-management programs may be enhanced by automated screening of LASA medicine name pairs. The software (LASA v2) is the first of its kind in Australia, and has shown its potential in the context of a national safety initiative, to identify confusable medicines. Healthcare services and pharmacies in Australia can use the software to identify confusable medicine name pairs relevant to their local setting. It will 
assist health services and pharmacies to make decisions when revising their formulary, and when implementing safety strategies to prevent LASA medicine errors.

We have successfully adapted the Food and Drug Administration's software, POCA, to use in the Australian setting, and used a novel approach to successfully design and test the usefulness of our software, LASA v2. Similar to processes in the USA and Canada, the current findings also point to the need to trial a process for Australian pharmaceutical manufacturers to screen their proposed medicine brand names, to reduce LASA medicine risks and avert further infiltration of the market with confusable medicine names.

While the input data (Australian Medicines Terminology) require transformation to compute similarity scores, and the output file requires some interpretation and elimination of falsepositive name matches, LASA $v 2$ offers a number of practical advantages over manual processes: efficiency compared to manual calculation of name similarity; inclusion of a second measure of orthographic similarity and a measure of phonetic similarity; and reduced reliance on retrospective clinical incident reports involving LASA medicines.

Collectively, these features warrant further application of this software to supplement realworld clinical reports and to prompt clinical debate about the contexts in which errors of medicine name confusion occur.

\section{AUTHORS' CONTRIBUTIONS}

LE conceptualized the research in consultation with HD and GS. CC produced and tested the software. LE trialled the software, with input from HD, GS and CC. LE drafted the 
manuscript. HD, GS and CC reviewed the manuscript. CC drafted Supplemental File 1. LE, HD and GS reviewed Supplemental File 1.

\section{ACKNOWLEDGEMENTS}

This work received financial and in-kind support from the Australian Commission on Quality and Safety in Health Care [grant number 58059]. The authors acknowledge critique of the software and outputs by the Commission's former Tall Man Lettering Expert Advisory Group and endorsement by the Health Services Medication Expert Advisory Group.

\section{DECLARATION OF INTERESTS}

Authors GS and HD are employees of the Australian Commission on Quality and Safety in Health Care. The software designed in this study has no commercial benefit to the Commission. Authors LE and CC have no interests to declare.

\section{SUMMARY TABLE}

\section{What was already known on the topic:}

- Look-alike, sound-alike medicines present risks for prescribing, dispensing and medication administration errors.

- Additional risks arise with increasing complexity of medicine names, approval of new entities and high-risk categories of medicines. 


\section{What this study added to our knowledge:}

- It is possible to screen a national register of medicines to compute medicine name similarity scores.

- Interpretation of name similarity scores, with consideration of the product presentation, proximity of storage and clinical usage, can be used to prioritize name pairs for riskmanagement initiatives.

- Automated screening of medicine name similarity may provide an efficient and proactive approach to alerting safety experts to risks of medicine name confusion.

\section{REFERENCES}

1. Rash-Foanio C, Galanter W, Bryson M, Falck S, Liu K, Schiff G, et al. Automated detection of look-alike/sound-alike medication errors. Am J Health-Syst Pharm 2017;74:5217.

2. Trbovich P, Hyland S. Responding to the challenge of look-alike, sound-alike drug names. BMJ Qual Saf 2017;26:357-9.

3. Her Q, Toh S. Sound-alike look-alike confusion and matching medication product attributes: simulated case-control studies. Ann Pharmacother 2019.

4. Lambert B, Galanter W, Liu K, Falck S, Schiff G, Rash-Foanio C, et al. Automated detection of wrong-drug prescribing errors. BMJ Qual Saf 2019;28:908-15.

5. Emmerton LM, Rizk MF. Look-alike and sound-alike medicines: risks and 'solutions'. Int J Clin Pharm 2012;34:4-8.

6. US Food and Drug Administration. How FDA reviews proposed drug names. US Food and Drug Administration. Available from: 
https://www.fda.gov/downloads/drugs/drugsafety/medicationerrors/ucm080867.pdf. Accessed 11 Nov 2019.

7. Government of Canada. Guidance document for industry - review of drug brand names. Government of Canada; 2014. Available from: https://www.canada.ca/en/healthcanada/services/drugs-health-products/reports-publications/medeffect-canada/guidancedocument-industry-review-drug-brand-names.html\#a21. Accessed 11 Nov 2019.

8. Therapeutic Goods Administration. Best practice guideline on prescription medicine labelling. Australian Government Department of Health; 2008. Available from: https://www.tga.gov.au/publication/best-practice-guideline-prescription-medicine-labelling. Accessed 11 Nov 2019.

9. MHRA. MHRA guideline for the naming of medicinal products and Braille requirements for name on label. Medicines and Healthcare products Regulatory Agency 2009; 2009. Available from:

https://assets.publishing.service.gov.uk/government/uploads/system/uploads/attachment_data /file/383713/Naming_of_medicines.pdf. Accessed 11 Nov 2019.

10. ASHP. USP says thousands of drug names look or sound alike. American Society of Healthsystem Pharmacists; 2008. Available from:

https://www.ashp.org/news/2008/02/13/usp_says_thousands_of_drug_names_look_or_sound _alike. Accessed 11 Nov 2019.

11. Australian Commission on Safety and Quality in Health Care. National Tall Man lettering list. Sydney, Australia: ACSQHC; 2016. Available from: https://www.safetyandquality.gov.au/our-work/medication-safety/safer-naming-labellingand-packaging-of-medicines/national-tall-man-lettering-list/. Accessed 11 Nov 2019.

12. Grissinger M. Tall Man letters are gaining wide acceptance. Pharmacy and Therapeutics 2012;37:132-3. 
13. Lambert B, Schroeder S, Galanter W. Does Tall Man lettering prevent drug name confusion errors? Incomplete and conflicting evidence suggest need for definitive study. $B M J$ Qual Saf 2016;25:213-7.

14. Filik R, Price J, Darker IT, Gerrett D, Purdy K, Gale A. The influence of Tall Man lettering on drug name confusion - a laboratory-based investigation in the UK using younger and older adults and healthcare practitioners. Drug Saf 2010;33:677-87.

15. Darker IT, Gerrett D, Fllik R, Purdy K, Gale A. The influence of 'Tall Man' lettering on errors of visual perception in the recognition of written drug names. Ergonomics 2011;54:21-33.

16. Campmans Z, Van Rhijn A, Dull R, Santen-Reestman J, Taxis K, Borgsteede S. Preventing dispensing errors by alerting for drug confusions in the pharmacy information system - a survey of users. PLoS ONE 2018;13:e0197469.

17. Australian Commission on Safety and Quality in Health Care. National standard for the application of Tall Man lettering: project report. Sydney, Australia: ACSQHC; 2011. Available from: http://www.safetyandquality.gov.au/wp-content/uploads/2013/03/NationalStandard-for-the-Application-of-Tall-Man-Lettering-Project-Report-with-appendices-PDF700KB.pdf. Accessed 11 Nov 2019.

18. US Food and Drug Administration. Phonetic and orthographic computer analysis (POCA) program. 2016. Available from:

http://www.fda.gov/Drugs/ResourcesForYou/Industry/ucm400127.htm. Accessed 11 Nov 2019.

19. Health Canada. Drug product database online query. Ontario, Canada: Government of Canada; 2018. Available from: https://health-products.canada.ca/dpd-bdpp/index-eng.jsp. Accessed 11 Nov 2019. 
20. Kondrak G, Dorr B. Automatic identification of confusable drug names. Artif Intell Med 2006;36:29-42.

21. Australian Digital Health Agency. National clinical terminology service. Canberra, Australia: Australian Government; 2016. Available from:

https://www.healthterminologies.gov.au/. Accessed 11 Nov 2019.

22. Therapeutic Goods Administration. Australian Register of Therapeutic Goods.

Canberra, Australia: Australian Government Department of Health. Available from: https://www.tga.gov.au/artg. Accessed 11 Nov 2019.

23. Australian Commission on Safety and Quality in Health Care. APINCHS classification of high risk medicines. Sydney, Australia: ACSQHC; 2017. Available from: https://www.safetyandquality.gov.au/our-work/medication-safety/high-riskmedicines/apinchs-classification-of-high-risk-medicines/. Accessed 11 Nov 2019.

24. Australian Commission on Safety and Quality in Health Care. High risk medicines. Sydney, Australia: ACSQHC; 2017. Available from:

https://www.safetyandquality.gov.au/our-work/medication-safety/high-risk-medicines/. Accessed 11 Nov 2019.

25. Australian Medicines Handbook Pty Ltd. Australian Medicines Handbook. Adelaide, Australia: AMH; 2019. Available from: https://amhonline.amh.net.au/auth. Accessed 11 Nov 2019.

26. Therapeutic Goods Administration. Updating medicine ingredient names - list of affected ingredients. Canberra, Australia: Australian Government Department of Health. Available from: https://www.tga.gov.au/updating-medicine-ingredient-names-list-affectedingredients. Accessed 11 Nov 2019.

27. Thompson CA. USP says thousands of drug names look or sound alike. Am J Health Syst Pharm 2008;65:386-8. 
28. Schroeder S, Salomon M, Galanter W, Schiff G, Vaida A, Gaunt M, et al. Cognitive tests predict real-world errors: the relationship between drug name confusion rates in laboratory-based memory and perception tests and corresponding error rates in large pharmacy chains. BMJ Qual Saf 2017;26:395-407.

29. Chan A, Muller R, Mancuso C. Description of a tall-man lettering program for lookalike and sound-alike antineoplastic agents. Am J Health-Syst Pharm 2018;75:512-3. 